\title{
Computed Tomography Measurement of 3D Combustion Chemiluminescence Using Single Camera
}

\author{
Kuanliang Wang, Fei Li*, Hui Zeng, Shaohua Zhang and Xilong Yu \\ State Key Laboratory of High Temperature Gas Dynamics, Institute of Mechanics, CAS, \\ No.15 Beisihuanxi Road, Beijing 100190, China
}

\begin{abstract}
Instantaneous measurement of flame spatial structure has been long desired for complicated combustion condition (gas turbine, ramjet et.). Three dimensional computed tomography of chemiluminescence (3D-CTC) is a potential testing technology for its simplicity, low cost, high temporal and spatial resolution. In most former studies, multi-lens and multi-CCD are used to capture projects from different view angles. In order to improve adaptability, only one CCD was utilized to build 3D-CTC system combined with customized fiber-based endoscopes (FBEs). It makes this technique more economic and simple. Validate experiments were made using 10 small $\mathrm{CH} 4$ diffusion flame arranging in a ring structure. Based on one instantaneous image, computed tomography can be conducted using Algebraic Reconstruction Technique (ART) algorithm. The reconstructed results, including the flame number, ring shape of the flames, the inner and outer diameter of ring, all well match the physical structure. It indicates that 3D combustion chemiluminescence could be well reconstructed using single camera.
\end{abstract}

Three dimensional computed tomography of chemiluminescence (3D-CTC), radicals, reconstruction, Algebraic Reconstruction Technique (ART)

\section{Introduction}

Chemiluminescence of flame are the emitted photons caused by the transition of excited-states radicals to ground-states. It is recognized as an important indictor in combustion diagnostics and control ${ }^{[1-3]}$. Compared with laser diagnostics, 3D-CTC is substantially simpler and easier to implement, also can provide information about key combustion quantities (e.g., heat release rate, local equivalence ratio) ${ }^{[4]}$, making it attractive for applications in harsh industrial environments. Chemiluminescence of flame have been studied for more than one century, and the first approach of measurement by designing a collection system to detect relatively small volume was demonstrated in $1991^{[5]}$. Cassegrain telescope optics subsequently have been utilized for non-intrusive measurement ${ }^{[6,7]}$. Multiple dimension measurement could be accomplished by rapid scanning the focusing plane if the target flame is steady. This method can not resolve the temporal dynamics of the combustion processes. The second approach for multiple dimension measurement is 3D-CTC technique combining chemiluminescence with tomography to obtain spatially-resolved measurements, which can provide more directly and critical structural information. Nonetheless, high spatial resolution and rapid temporal resolution measurement is a tremendous challenge for 3D-CTC technique due to the limits of optical access and hardware. In recent years, 3D-CTC have been widely studied due to the great improvements of capabilities of optical sensing and computing technologies. Based on different imaging system, 3D-CTC can be divided into three categories ${ }^{[3]}$. The first category obtains the image projection by custom-made lens and film ${ }^{[8]}$, the second method based on lens and CCD cameras ${ }^{[4,9,10]}$, and the third method combined fiber-based endoscopes(FBEs) with multi-CCD to get the multi-direction projections ${ }^{[11,12]}$.

\footnotetext{
* Corresponding author: lifei@imech.ac.cn
} 
Using customized FBEs, this method can cut down the number of CCD making the technique more economic and simple. Besides, most 3D-CTC reconstructions are based on the traditional model of parallel beam assumption, which does not fit in with the light collection of optical system, especially for FBEs.

Current work focuses on approaches of FBEs based 3D-CTC, particularly the imaging process. Lens imaging theory was used to deduce the distribution of voxels on $\mathrm{CCD}^{[3]}$. And parameters, including distances and angles, were calibrated to calculate the weight coefficients. Algebraic Reconstruction Technique (ART) algorithm ${ }^{[13]}$ was used To solve the tomographic problem. The reconstruction program was also validated numerical through phantoms with Gaussian distribution, and the reconstruction accuracy was analyzed at different viewing angle and imaging noise. For better adaptability, only one CCD was utilized combined with customized FBEs, which has 9 inputs and 1 outputs. Validate experiments were made using 10 small CH4 diffusion flame arranging in a ring structure. Results show that 3D combustion chemiluminescence could be well reconstructed using single camera.

\section{Fundamentals of reconstruction by projections}

The physical formation of the CTC problem is shown in Fig.1, where the flame is located in the cubical object domain. If we use $F(x, y, z)$ to denote the 3D distribution of the chemiluminescence emission signals emitted from object domain, the projection measured $\left(I_{p}\right)$ from a viewing angle $(q)$ can then be defined in Eq. 1.

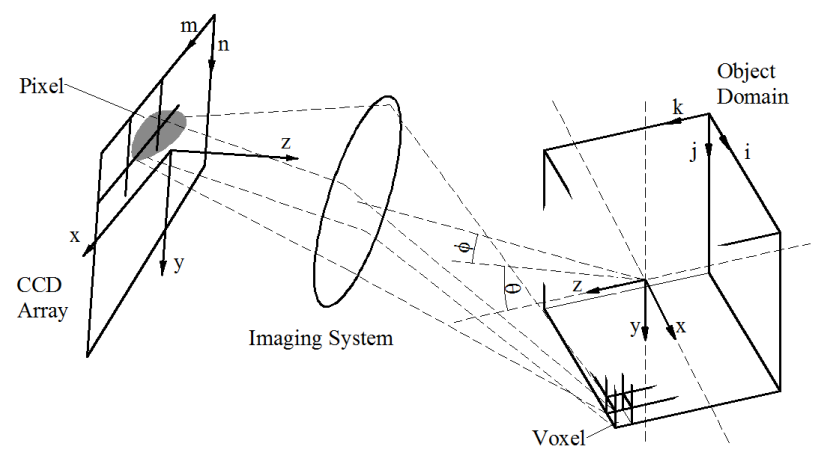

Fig.1 Mathematical formulation of 3D-CTC

$$
I_{p q}=\int_{p q} F(x, y, z) d v
$$

Here, the viewing angles can be specified by $\mathrm{r}$ (distance), $\theta$ (azimuth angle), and $\phi$ (inclination angle), and the projection refers to a single integral measurement of a pixel (index p) on the camera chip. it is a weighted summation of all voxels. Mathematically, the distribution problem was solved by discretizing $F$ into voxels in the Cartesian coordinate system as show, and therefore we can rewrite Eq. 1 in the matrix form:

$$
\boldsymbol{I}=\boldsymbol{W} \times \boldsymbol{F}
$$

In this equation the element of $\boldsymbol{W}$ represents the signal each pixel captured from each voxel, and $\boldsymbol{I}$ is the weighted summation of all voxels, so we named $\boldsymbol{W}$ as weight matrix. Weight matrix represents the relationship between pixel and the corresponding position of objective region.

There are various iterative algorithms for the tomographic, and the most widely used one is Algebraic Reconstruction Technique(ART). It was firstly developed by Gordon ${ }^{[13]}$ to solve the $3 \mathrm{D}$ reconstruction problem from 
projection of electron microscopy and radiology, and has been modified into many different forms. Here we utilized an additive version of ART, which has been adopted by Flord ${ }^{[9]}$ :

$$
F^{(k+l)}=F^{(k)}+\lambda \bullet \boldsymbol{W} \frac{I_{p q}-\boldsymbol{W} F^{(k)}}{\boldsymbol{W} \cdot \boldsymbol{W}}
$$

Based on the physical formation and mathematical formulation demonstrated above, we developed a multi-directional tomography reconstruction program. Before dealing with experimental images, this program was validated numerical through phantoms with Gaussian distribution, and the comparison between reconstruction error and the number of views is analyzed.

\section{Numerical validation}

Gaussian function was used to assume the 3D distribution of the object domain, and then projections can be calculated through phantoms. Reconstructed distributions by different number of views were compared with the phantoms to validate the veracity of the program. As shown in Fig. 2, the object domain was discrete as $30 * 30 * 30$ voxels, and the middle layer of the vertical direction was chosen for the comparison. Reconstructed results are shown in Fig.2(a-d), corresponding 3, 5,7 and 9 number of views, respectively.

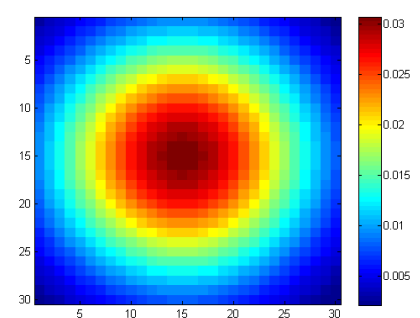

phantom

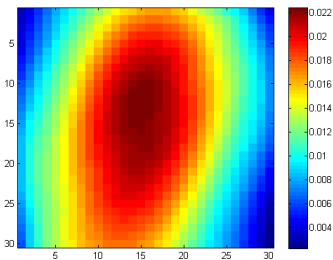

(a)

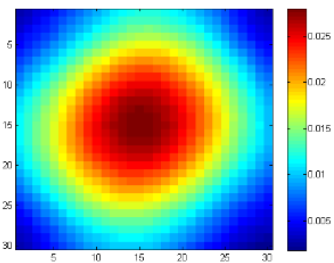

(b)

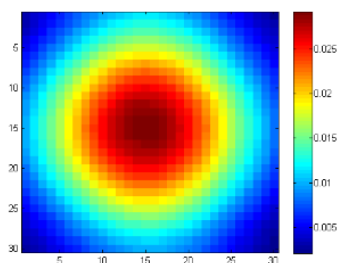

(c)

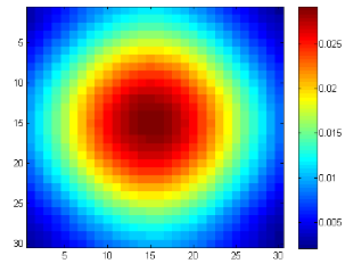

(d)

Fig. 2 Comparison between phantom and reconstructions by 3(a), 5(b),7(c), 9(d) views

As can be seen, a positive correlation exists between accuracy and viewing number. When we use views less than 5 to reconstruct the distribution, there is a big difference between the reconstruction and phantom. To quantify the reconstruction accuracy, the inversion error is defined by the following equation:

$$
\text { error }=\frac{\sum_{i} \sum_{j} \sum_{k}\left|F^{r e c}(x, y, z)-F(x, y, z)\right|}{\sum_{i} \sum_{j} \sum_{k}|F(x, y, z)|}
$$

Here $F^{r e c}$ is the distribution of reconstruction, and $F$ is the phantom. For the interference of noise is unavoidable in the experiment process, we also analyze the inversion error of different noise level. The results are 
demonstrated in Fig.3. The decreasing error indicates that the better performance of more views available. And views more than 8 should be utilized for adequate accuracy. In addition, these noises level of $5 \%$ does not bring an obvious deterioration of reconstruction accuracy.

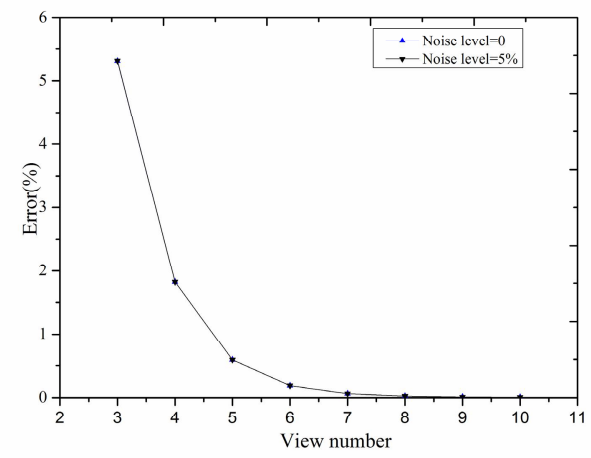

Fig.3 inversion error for different noise level and view numbers

\section{Experimental apparatus and condition}

Multi-directional imaging system is achieved by the setups shown in Fig.4. Target flames were 10 small CH4 diffusion flame arranging in a ring structure. As shown in Fig.4(b), the inner and outer diameter of the flames were $25.18 \mathrm{~mm}$ and $37.74 \mathrm{~mm}$, respectively. As shown in (c), the size of each flame was slightly bigger than the diameter of the single burner exit, which was about $5.2 \mathrm{~mm}$. Customized FBEs was used to achieve 9 views. It has 9 inputs and only one outputs, therefore, single camera was used to capture 9 views simultaneously. Each inputs is an assembling of 13,000 fibers, so the output end transits 117,000 image elements to CCD. The pixel size of CCD (IMI 147FT) is 6.45um*6.45um, and images were taken with exposure time of 100us during this experiments. As shown in Fig.4, XY plane is vertical view for object domain, and the projection got from 9 number of views (inputs of the FBEs) around the burner plane. The parameters of each view angle include distance, rotation angle, pitch angle, the spin angle of FBEs input and the offset of optical axis on the XY plane (specified by Tx and Ty). In order to calibrate these parameters, many luminescent spots produced by a He-Ne laser were used. The calibrated results are listed in Table 1.
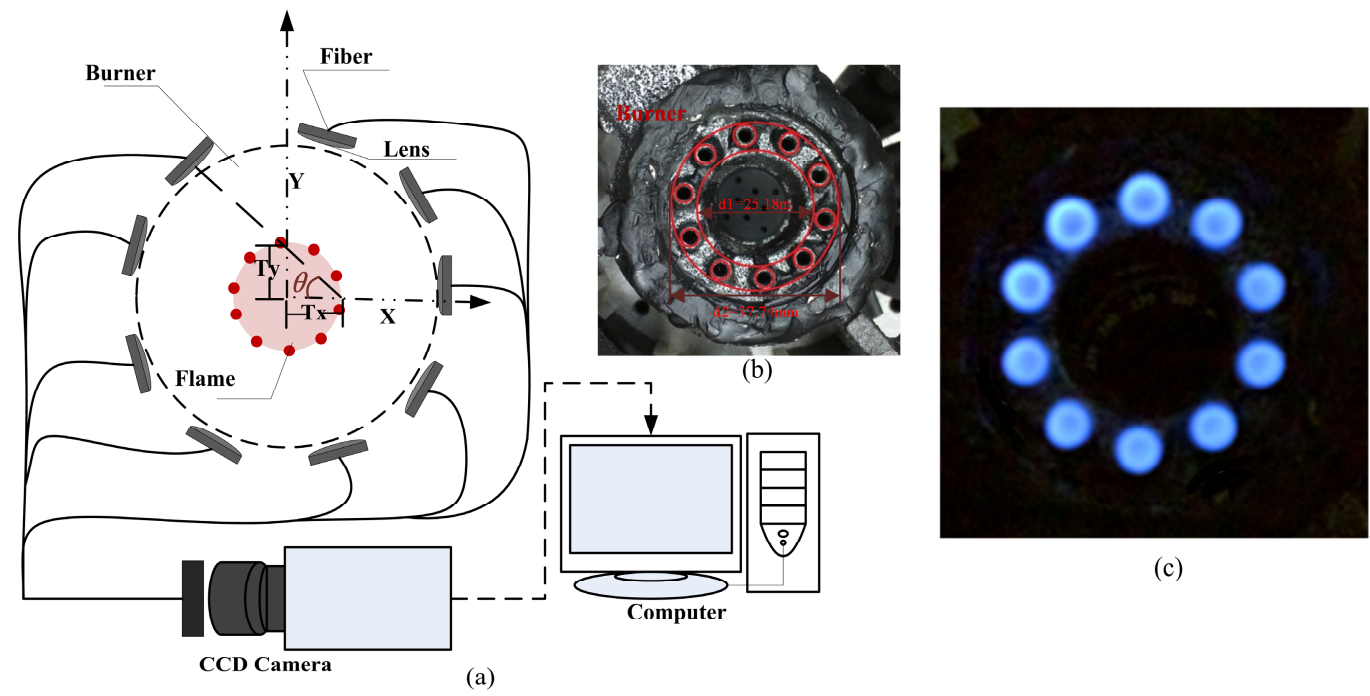

(c)

Fig.4 3D-CTC projection measurement system 
Table 1 view angles of 9 fibers

\begin{tabular}{ccccccc}
\hline $\begin{array}{c}\text { View } \\
\text { number }\end{array}$ & $\begin{array}{c}\text { Rotation } \\
{\left[^{\circ}\right]}\end{array}$ & $\begin{array}{c}\text { Pitch } \\
{\left[^{\circ} \mathbf{]}\right.}\end{array}$ & $\begin{array}{c}\text { Spin } \\
{\left[^{\circ}\right]}\end{array}$ & $\begin{array}{c}\text { Distance } \\
{[\mathbf{m m}]}\end{array}$ & $\begin{array}{c}\text { Tx } \\
{[\mathbf{m m}]}\end{array}$ & $\begin{array}{c}\text { Ty } \\
{[\mathbf{m m}]}\end{array}$ \\
\hline $\mathbf{1}$ & 126.25 & 7.60 & 91.69 & 67.44 & -9.95 & 1.05 \\
$\mathbf{2}$ & 74.79 & 13.81 & 18.87 & 71.87 & 10.08 & 2.23 \\
$\mathbf{3}$ & 42.53 & 9.17 & 111.52 & 67.97 & -10.07 & 2.51 \\
$\mathbf{4}$ & 11.09 & 13.71 & -88.15 & 66.76 & -9.75 & 3.34 \\
$\mathbf{5}$ & 335.22 & 16.49 & -79.99 & 63.39 & -8.45 & 4.16 \\
$\mathbf{6}$ & 288.86 & 13.17 & -45.15 & 61.71 & -4.89 & 2.96 \\
$\mathbf{7}$ & 226.83 & 14.14 & -87.40 & 63.15 & -9.97 & -2.27 \\
$\mathbf{8}$ & 185.60 & 15.68 & 33.25 & 73.46 & 2.69 & 8.40 \\
$\mathbf{9}$ & 153.22 & 13.64 & 25.31 & 75.94 & 2.52 & 6.36 \\
\hline
\end{tabular}

\section{Experimental results}

The raw single-shot image of the flames is shown in Fig.5, corresponding to 9 views. No smooth was made during data processing. Reconstructed object size was $45^{*} 45^{*} 15 \mathrm{~mm}$, slightly larger than the flames. It was discretized into $120 * 120 * 30$ in $\mathrm{X}, \mathrm{Y}, \mathrm{Z}$ directions, so there are 432,000 voxels and each has a size of $0.375 * 0.375 * 0.5 \mathrm{~mm}$. Utilized the former program, the reconstructed results are shown in Fig.6 and Fig.7. Fig6 is the sum of all layers along z direction. It can be used to compare to the top view of flames shown in Fig.4(c). We can firmly see 10 small flames arranging in a ring structure. The diameter of inner circle is about 70 voxels, and the outer circle is about 110 voxels, which well match Fig4.(b) and (c).

Fig7 is the 2D section of the reconstructed 3D flame distribution, and all the sections parallel to XY plane. From the distribution on different layer we can calculate the thickness of the flames, which is about $5.5 \mathrm{~mm}$. We can also find that there has a small inclination between the XY plane and the flames. It also meet the physical arrange of the burner. All these results indicate that this image system can reconstruct 3D combustion chemiluminescence.
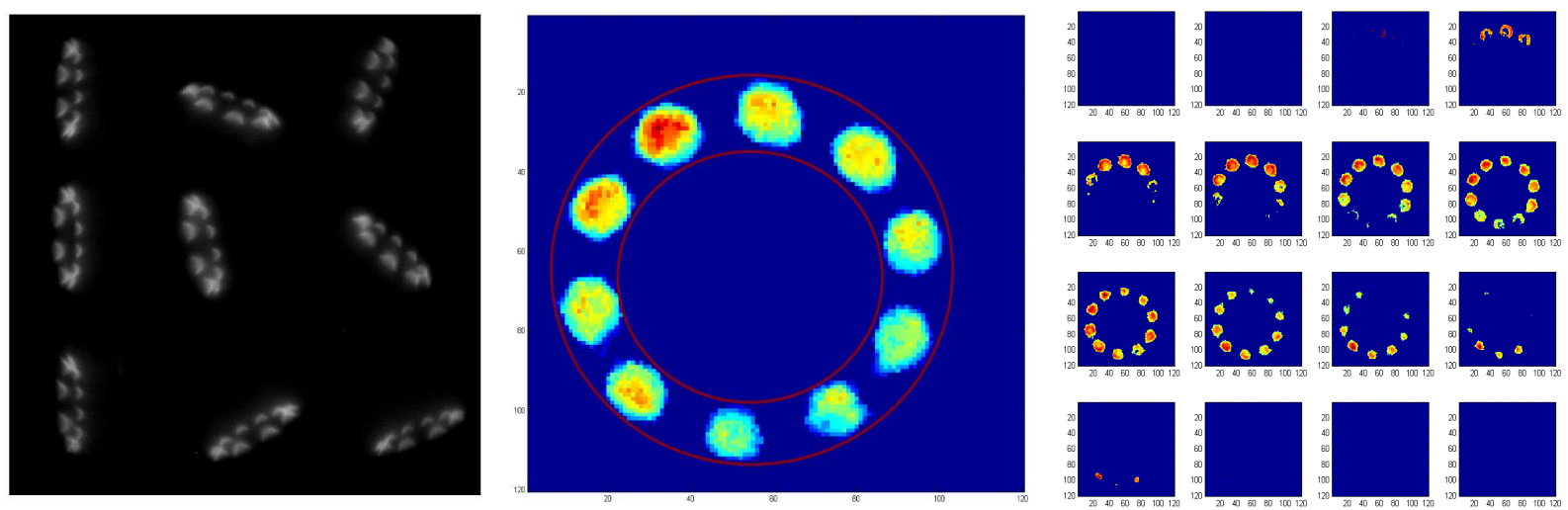

Fig.5 Simultaneous projections from 9 fibers Fig.6 Sertical view of reconstructed flame

Fig.7 Sross-sections of reconstructed flame 


\section{Summary}

3D-CTC were achieved using single camera and fiber-based endoscopes. Both numerical and experimental validation were conducted to study the capacity of our reconstruction system. Based on error analysis of the numerical validation, at least 8 views should be used for adequate accuracy. A ring burner with 10 small CH4 diffusion flame was used as target object, 3D reconstruction results from one instantaneous image shows each flame size and the ring diameter. It estimates the accuracy and resolution of this single camera system.

\section{Acknowledgment}

This work was sponsored by the National Natural Science Foundation of China (Grant Nos. 11372329).

\section{Reference}

[1] Kathrotia, T., Riedel, U., Seipel, A., Moshammer, K., Brockhinke, A., "Experimental and numerical study of chemiluminescent species in low-pressure flames",Appl. Phys. B: Lasers Opt. 107(3),571-84 (2012).

[2] Anikin, Nb, Suntz, R, Bockhorn, H, "Tomographic reconstruction of 2D-OH*-chemiluminescence distributions in turbulent diffusion flames",Appl. Phys. B. 107(3),591-602 (2012).

[3] Wang, J., Song, Y., Li, Z. H., Kempf, A., He, A. Z., "Multi-directional 3D flame chemiluminescence tomography based on lens imaging",Opt. lett. 40(7),1231-4 (2015).

[4] Cai, Weiwei, Li, Xuesong, Li, Fei, Ma, Lin, "Numerical and experimental validation of a three-dimensional combustion diagnostic based on tomographic chemiluminescence",Opt. express. 21(6),7050-64 (2013).

[5] Nakabe, Kazuyoshi, Mizutani, Yukio, Hirao, Tomoyuki, Fujioka, Hiroyuki, "An experimental study on detailed flame structure of liquid fuel sprays with and without gaseous fuel",Combust. Flame. 84(1-2),3-14 (1991).

[6] Aleiferis, P. G., Hardalupas, Y., Taylor, A. M. K. P., Ishii, K., Urata, Y., "Flame chemiluminescence studies of cyclic combustion variations and air-to-fuel ratio of the reacting mixture in a lean-burn stratified-charge spark-ignition engine",Combust. Flame. 136(1-2),72-90 (2004).

[7] Hardalupas, Y., Panoutsos, C. S., Taylor, A. M. K. P., "Spatial resolution of a chemiluminescence sensor for local heat-release rate and equivalence ratio measurements in a model gas turbine combustor ",Exp. Fluids. 49(4),883-909 (2010).

[8] Ishino, Y, Takeuchi, K, Shiga, S, Ohiwa, N. "Measurement of instantaneous 3D-distribution of local burning velocity on a turbulent premixed flame by non-scanning 3D-CT reconstruction",proceedings of the 4th European Combustion Meeting, Vienna, Austria, April, 14-17 (2009).

[9] Floyd, J., Geipel, P., Kempf, A. M., "Computed Tomography of Chemiluminescence (CTC): Instantaneous 3D measurements and Phantom studies of a turbulent opposed jet flame",Combust. Flame. 158(2),376-91 (2011).

[10] Floyd, J., Kempf, A. M., "Computed Tomography of Chemiluminescence (CTC): High resolution and instantaneous 3-D measurements of a Matrix burner",P. Combust. Inst. 33(1),751-8 (2011).

[11] Kang, Minwook, Wu, Yue, Ma, Lin, "Fiber-based endoscopes for 3D combustion measurements: View registration and spatial resolution",Combust. Flame. 161(12),3063-72 (2014).

[12] Ma, Lin, Lei, Qingchun, Wu, Yue, Ombrello, Timothy M., Carter, Campbell D., "3D measurements of ignition processes at $20 \mathrm{kHz}$ in a supersonic combustor", Appl. Phys. B. 119(2),313-8 (2015).

[13] Gordon, Richard, "A tutorial on ART (algebraic reconstruction techniques)", IEEE Trans. Nucl. Sci.,. 21(3),78-93 (1974). 